Short Note

\title{
Regularization of reaction progress variable for application to flamelet-based combustion models
}

\author{
Matthias Ihme ${ }^{\mathrm{a}, *}$, Lee Shunn ${ }^{\mathrm{b}}$, Jian Zhang ${ }^{\mathrm{c}}$ \\ ${ }^{a}$ Department of Aerospace Engineering, University of Michigan, Ann Arbor, MI 48109, United States \\ ${ }^{\mathrm{b}}$ Cascade Technologies Inc., Palo Alto, CA 94303, United States \\ ${ }^{\mathrm{c}}$ LNM, Institute of Mechanics, CAS, Beijing 100190, China
}

\section{A R T I C L E I N F O}

\section{Article history:}

Received 3 February 2012

Received in revised form 1 June 2012

Accepted 21 June 2012

Available online 4 July 2012

\section{Keywords:}

Reaction progress variable

Combustion model

Flamelet model

\begin{abstract}
A B S T R A C T
Many combustion models that are based on the flamelet paradigm employ a reaction progress variable. While such a progress variable is well defined for one-step reaction kinetics, this is typically not the case for complex chemical mechanisms. Consequently, several expressions for a progress variable have been utilized. In this paper a formal method for the generation of a reaction progress variable is proposed that is optimal with respect to a set of constraints. The potential of the method is demonstrated in applications to partially premixed and diffusion flames, and the extension to premixed combustion is discussed. It is shown that the proposed method can lead to significant improvements in the definition of an optimal progress variable over conventional formulations, essentially eliminating the expert knowledge previously required in identifying such quantities.
\end{abstract}

(C) 2012 Elsevier Inc. All rights reserved.

\section{Introduction}

Detailed kinetic mechanisms for combustion can comprise thousands of chemical reactions amongst hundreds of molecular species [1]. Despite significant advances in the power and availability of computational resources, numerical simulations of realistic combustion problems remain prohibitively expensive due to the large number of species and the disparate range of important chemical and flow scales present. To overcome this challenge, several methods have been developed which project the high-dimensional thermochemical state-space onto a low-dimensional manifold. In this approach, the entire state-space is pre-computed and parameterized in terms of a reduced set of scalar variables that are conveniently computed in a combustion simulation, thus providing efficient access to detailed thermochemistry at a greatly reduced computational expense. These thermochemical manifolds are typically parameterized using a combination of conserved and reactive scalars. Generally speaking, the chemical composition of the fully-reacted mixture is determined from conserved quantities (such as mixture fraction $Z$ or equivalence ratio $\phi$ ), while the progress of combustion between unburned and burned states is described by reactive scalars, which are also referred to as reaction progress variables.

In current combustion models, the progress variable (which is hereafter referred to as $C$ ) is often defined based on the experience and intuition of the user. Consequently, a universally accepted definition is not available. In this paper, a general framework for the identification of a realizable reaction progress variable is proposed. This general expression can significantly reduce possible ambiguities that are often associated with the selection of an appropriate set of species from which $C$ is subsequently defined. It is anticipated that this new method for specifying $C$ will prove useful in novel combustion applications, such as low-temperature combustion, combustion with multiple reactant streams, and new fuel compositions, for which only limited experience is available.

\footnotetext{
* Corresponding author.

E-mail address: mihme@umich.edu (M. Ihme).
} 


\subsection{Overview of relevant combustion models}

Different approaches have been proposed to represent combustion chemistry in numerical simulations. One popular method is the laminar flamelet model, which views a turbulent flame as an ensemble of thin, quasi-laminar reaction zones embedded within a turbulent flow field [2,3]. This assumption, based on physical arguments about the relevant length and time scales, allows for a simplified description of the interaction between the chemistry and the hydrodynamics of the flow. The chemistry is represented separately by computing a series of one-dimensional laminar flames, each subjected to varying amounts of hydrodynamic strain (in the case of diffusion flames) or equivalence ratios (for premixed flames). Therefore, the solutions that are obtained from the steady flamelet equations lie on a two-dimensional manifold, and can be characterized by the mixture composition and the extent of reaction.

Another powerful approach to simplify combustion chemistry is the intrinsic low-dimensional manifold (ILDM) method of Maas \& Pope [4]. ILDM uses tools from dynamical systems theory to automatically analyze and reduce complex kinetic mechanisms. This is accomplished by using the eigenvalues of the reaction system to distinguish between fast and slow chemical processes and to identify attracting manifolds in composition space. Although ILDM produces a mathematically rigorous description of the chemical system, accurate representations of the low- and mid-temperature behavior of basic hydrocarbon fuels typically require the specification of manifolds with at least four or five dimensions. As ILDM uses a direct mathematical analysis of the dynamic response of the chemical system, it ultimately produces a tabulation of the chemistry that is applicable in any combustion regime (premixed, partially-premixed, or non-premixed).

Recently, investigators have combined some of the benefits of ILDM with flamelet-based models in order to improve the global accuracy of the chemical description while reducing the dimensionality of the parameter space. Such methods include the flame-prolongation of ILDM (FPI) [5], flamelet-generated manifolds (FGM) [6], and the flamelet/progress-variable (FPV) model $[7,8]$. The utility of these methods has been demonstrated in a variety of problems, including laminar premixed flames [5,6,9,10], laminar partially-premixed flames [11,12], laminar diffusion flames [12], turbulent premixed burners [13], and turbulent non-premixed systems $[7,8,14,15]$.

Although the FPI, FGM, and FPV models have substantial differences, there are many similarities in the way that they are framed and implemented. In each method, a manifold is constructed by solving equations for steady, one-dimensional laminar flames. Various parameters in these "flamelet" equations (such as reactant conditions, mass burning rate, flame stretch, strain rate, etc.) are systematically varied in order to produce a collection of laminar flame states that describe the gamut of conditions that will be encountered in the simulation. The flamelet solutions form a chemistry database that is parameterized by relevant controlling variables and accessed by the flow solver during combustion simulations via table-lookups. Each of these models uses a reaction progress variable as one of the input parameters to the tabulation.

To develop the method, an adiabatic two-stream combustion system is considered, for which the thermochemical manifold is parameterized in terms of mixture fraction $Z$ and reaction progress variable $C$. This state-space representation can be written in the form

$$
\psi=\psi(Z, C),
$$

where $\psi$ is the vector of all species mass fractions, temperature, chemical source terms, and viscous-diffusive properties. While $Z$ is an intrinsic independent parameter in the flamelet formulation, the selection of the progress variable as a second independent coordinate that uniquely parameterizes $\psi$ is a concern that is addressed in this paper.

\subsection{Progress variable and the role of a flamelet parameter}

The progress variable $C$ is commonly defined from a combination of reactive scalars such as chemical species or temperature. Although its definition is not unique, the choice of a suitable progress variable should be guided by the following principles:

(a) The definition of $C$ should result in a transport equation that can be conveniently solved in a combustion simulation.

(b) The reactive scalars from which $C$ is constructed should all evolve on comparable time scales.

(c) All parameters that define the manifold should be independent of one another.

(d) The set of parameters from which the manifold is formed should uniquely characterize each point in the thermochemical state-space.

Various definitions for the progress variable have been employed. For example, the following expressions have been used as progress variables in applications of hydrocarbon combustion:

$$
\begin{aligned}
& \text { Pierce \& Moin [7] : } \quad C=Y_{\mathrm{CO}_{2}}+Y_{\mathrm{H}_{2} \mathrm{O}}, \\
& \text { Ihme \& Pitsch }[14,15]: \quad C=Y_{\mathrm{CO}_{2}}+Y_{\mathrm{H}_{2} \mathrm{O}}+Y_{\mathrm{CO}}+Y_{\mathrm{H}_{2}} \text {, } \\
& \text { Fiorina et al. }[11,12,13]: C=Y_{\mathrm{CO}_{2}}+Y_{\mathrm{CO}} \text {, } \\
& \text { van Oijen \& de Goey [10]: } C=\frac{Y_{\mathrm{CO}_{2}} / M_{\mathrm{CO}_{2}}+Y_{\mathrm{H}_{2} \mathrm{O}} / M_{\mathrm{H}_{2} \mathrm{O}}+Y_{\mathrm{H}_{2}} / M_{\mathrm{H}_{2}}}{Y_{\mathrm{CO}_{2}}^{\mathrm{eq}} / M_{\mathrm{CO}_{2}}+Y_{\mathrm{H}_{2} \mathrm{O}}^{\mathrm{eq}} / M_{\mathrm{H}_{2} \mathrm{O}}+Y_{\mathrm{H}_{2}}^{\mathrm{eq}} / M_{\mathrm{H}_{2}}}
\end{aligned}
$$


where $Y_{i}$ and $M_{i}$ denote the mass fraction and molecular weight of species $i$, respectively, and the superscript "eq" in Eq. (C4) refers to the equilibrium composition. The progress variable is frequently normalized in some fashion such that $C \in[0,1]$.

All of the different progress variable representations, shown in Eqs. (C1)-(C4), fulfill principles (a) and (b). In all cases, the progress variable is formed from a linear combination of reactive scalars. Under the assumption of equal species diffusivities, this results in a clearly defined transport equation for the progress variable that can be conveniently modeled in a combustion simulation. Furthermore, all expressions for $C$ are primarily defined from major product species that are generated through reaction pathways which evolve on similar time scales.

To enforce independence amongst the variables that span the lower-dimensional manifold (principle (c)), a so-called flamelet parameter, denoted here by $\Lambda$, is introduced. Although the introduction of $\Lambda$ might initially appear to be a dispensable intermediate step, this is not the case. A clearly defined $\Lambda$ tightens the theoretical and notational framework for constructing a regularized reaction progress variable.

To emphasize the fundamental difference between $C$ and $\Lambda$, consider a flamelet-based combustion model that utilizes the two-dimensional manifold (1). Since in this case all of the species mass fractions are dependent on $Z$, it is apparent that $C=C(Z)$ which violates principle (c). While this may not be of direct relevance for laminar combustion applications, enforcing property (c) can lead to significant model simplifications for turbulent combustion applications [8,14,15].

Different approaches can be taken to construct the flamelet parameter $\Lambda$. For example, in the FPV-model, which is based on the steady non-premixed flamelet paradigm, the progress parameter is defined as [8]:

$$
\Lambda=C \mid Z_{\text {st }},
$$

corresponding to the stoichiometric value of the progress variable of each flamelet that constitutes the manifold. As such, $\Lambda$ should be understood as a parameter that uniquely identifies each flamelet solution. Similarly, $\Lambda$ could alternatively be computed as

$$
\Lambda=\int\left[\left(\frac{d T_{\mathrm{st}}}{d \chi_{\mathrm{st}}}\right)^{2}+1\right]^{1 / 2} \mathrm{~d} \chi_{\mathrm{st}},
$$

defining $\Lambda$ as the arc length along the S-shaped curve of the steady flamelet solutions. In this equation, $T$ is the temperature, $\chi$ is scalar dissipation rate, and the subscript "st" refers to the stoichiometric condition.

Note, that these definitions of $\Lambda$ result in rather intricate transport equations [16,17] that are difficult to model, violating principle (a). Therefore, $\Lambda$ is eventually replaced by $C$ during the pre-processing and tabulation of the data. This requires that $C$ be a strictly monotonic function of $\Lambda$ in order to produce a bijective mapping of the state-space. Furthermore, it ensures that all thermochemical states are uniquely represented by the parameter manifold, so that principle (d) is automatically fulfilled.

\section{Optimal reaction progress variable}

In developing a method for constructing an optimal progress variable, we consider a two-dimensional manifold in which all thermodynamic quantities are parameterized in terms of mixture fraction $Z$ and progress parameter $\Lambda$. The derivation begins by defining the reaction progress variable as a weighted linear combination of species mass fractions $Y_{i}$ [16]:

$$
C=\sum_{i \in \mathcal{S}} w_{i} Y_{i}(Z, \Lambda)
$$

where $w_{i}$ denotes the weighting coefficient for species $i$ that is contained in a predefined set of species $\mathcal{S}$. As example, for the specific case given in Eq. (C2), this set is $\mathcal{S}=\left\{\mathrm{CO}_{2}, \mathrm{CO}, \mathrm{H}_{2} \mathrm{O}, \mathrm{H}_{2}\right\}$, and Eq. (C2) is recovered by setting $w_{i}=1$ for all $i$ 's. The choice of species that are contained in $\mathcal{S}$ is made by the user, and can be guided by experience, sensitivity studies, or by a reaction time scale analysis $[18,19]$.

Whereas in previous applications the weight coefficients are typically assigned to be unity, here we will consider these weights as free parameters. The values for these coefficients are then adjusted to obtain a reaction progress variable that is optimal with respect to a user-defined cost function. From Eq. (4) it follows that $C=f(Z, \Lambda)$. The objective, then, is to find a progress variable such that $\Lambda=f^{-1}(Z, C)$ exists, allowing for the elimination of $\Lambda$ and the representation of all other thermochemical quantities in terms of $Z$ and $C$. For this inversion to be defined, $C$ and $\Lambda$ must be bijective. This constraint can be formulated as

$$
\partial_{\Lambda} C>\varepsilon \geqslant 0 .
$$

From this strict monotonicity requirement, a cost function $\mathcal{L}$ can be defined as:

$$
\mathcal{L}=\iint \Omega \mathcal{H}\left(\varepsilon-\partial_{\Lambda} C\right) d Z d \Lambda
$$

where $\mathcal{H}$ is the Heaviside function, and the penalty term $\Omega \in \mathbb{R}_{0}^{+}$is introduced as an additional weight function. For instance, by setting $\Omega=C$ particular emphasis is assigned to regions with large values of $C$, typically corresponding to chemically active zones in the thermochemical state-space. 
With the definition of $\mathcal{L}$, an optimization problem may be formulated which identifies the set of weight coefficients that minimizes the cost function:

$$
\begin{array}{ll}
\min _{\mathbf{w} \in \mathbb{R}^{|\mathcal{S}|}} & \mathcal{L} \\
\text { subject to } & i \in \mathcal{S} .
\end{array}
$$

To solve the unconstrained optimization problem, the search space is restricted so that $\mathbf{w} \in[-1,1]^{|\mathcal{S}|}$, and the weight coefficients are subsequently normalized so that $C \in[0,1]$.

Standard optimization methods can be utilized to solve Eq. (7), and in the following a derivative-free pattern search method [20] is employed. This method generates a sequence of iterates, whose cost function is non-increasing [21]. The algorithm consists of a search step which is followed by a poll step. While the search step assists in the global exploration of the parameter space, the poll step ensures convergence with respect to a mesh-local neighborhood. More details to the algorithm and its convergence properties can be found in Ref. [22].

\section{Application}

In the following, the method for constructing an optimal reaction progress variable is demonstrated in applications to partially premixed and diffusion flames. To this end, the steady laminar flamelet equations [3] are solved [23] to generate a database of one-dimensional flame-structures. The steady flamelet equations can be derived from an asymptotic analysis of the governing equations for species and temperature conservation [24]. Formulated in vector form this equation can be written as

$$
-\frac{\chi_{Z}}{2} \partial_{Z}^{2} \psi=\dot{\omega}
$$

where $\chi_{Z}=2 \alpha|\nabla Z|^{2}$ is the scalar dissipation rate, and $\dot{\omega}$ corresponds to the source term of all species and temperature, which are collectively denoted by the vector $\psi=\left(\mathbf{Y}^{T}, T\right)^{T}$. The database is then generated by solving Eq. (8) for a range of scalar dissipation rates, and parameterizing the resulting flamelet structures in terms of $Z$ and $\Lambda$.

In sequence of increasing fuel-complexity, we will first consider a methane/air configuration, and then extend this application to an $n$-heptane/air combustion system that is diluted by increasing amounts of hydrogen to investigate the effects of changing mixture composition on the definition of an optimal reaction progress variable.

\subsection{Methane/air combustion system}

The first application considers the oxidation of methane and diluents of air and hydrogen. The mixture compositions of the oxidizer and fuel streams are specified in Table 1. The chemistry is described by the GRI 2.11 mechanism [25], consisting of 279 reactions among 49 species. For this optimization problem, Eq. (3) was used to define the flamelet parameter. The normalized progress variable was used as weighting function $\Omega$ in Eq. (6), and the threshold value was set to $\varepsilon=1 \times 10^{-10}$ for all cases considered. For each of the flame configurations, three progress variables are constructed that are composed of different species. The choice of the species that are contained in the set $\mathcal{S}$ was motivated by the definitions given in Eq. (C1)-(C3). This allows us to compare different options and provide guidance as to which species should be considered in the definition of the reaction progress variable.

Table 1

\begin{tabular}{|c|c|c|}
\hline $\mathcal{S}$ & $\mathbf{w}$ & $\mathcal{L}$ \\
\hline \multicolumn{3}{|c|}{ Oxidizer $X_{\mathrm{O}_{2}}=0.21, X_{\mathrm{N}_{2}}=0.79 ;$ Fuel: $\mathrm{X}_{\mathrm{CH}_{4}}=1$} \\
\hline$\left\{\mathrm{CO}_{2}, \mathrm{CO}\right\}$ & $(0.635,0.648)^{T}$ & $4.374 \times 10^{-2}\left(4.681 \times 10^{-2}\right)$ \\
\hline$\left\{\mathrm{H}_{2} \mathrm{O}, \mathrm{CO}_{2}, \mathrm{CO}, \mathrm{H}_{2}\right\}$ & $(-0.766,0.860,0.883,0.972)^{T}$ & $1.605 \times 10^{-3}\left(8.688 \times 10^{-2}\right)$ \\
\hline \multicolumn{3}{|c|}{ Oxidizer: $X_{O_{2}}=0.21, X_{N_{2}}=0.79 ;$ Fuel: $X_{C_{b o l d 4}}=0.25, X_{O_{2}}=0.158, X_{N_{2}}=0.592$} \\
\hline$\left\{\mathrm{CO}_{2}, \mathrm{H}_{2} \mathrm{O}\right\}$ & $(0.360,-0.018)^{T}$ & $1.033 \times 10^{-2}\left(1.718 \times 10^{-2}\right)$ \\
\hline$\left\{\mathrm{CO}_{2}, \mathrm{CO}\right\}$ & $(0.457,0.717)^{T}$ & $5.971 \times 10^{-3}\left(9.290 \times 10^{-3}\right)$ \\
\hline$\left\{\mathrm{H}_{2} \mathrm{O}, \mathrm{CO}_{2}, \mathrm{CO}, \mathrm{H}_{2}\right\}$ & $(-0.281,0.391,0.259,0.627)^{T}$ & $3.297 \times 10^{-3}\left(1.381 \times 10^{-2}\right)$ \\
\hline \multicolumn{3}{|c|}{ Oxidizer: $X_{O_{2}}=0.21, X_{N_{2}}=0.79 ;$ Fuel: $X_{C_{4}}=0.5, X_{H_{2}}=0.5$} \\
\hline$\left\{\mathrm{CO}_{2}, \mathrm{H}_{2} \mathrm{O}\right\}$ & $(0.652,-0.016)^{T}$ & $6.660 \times 10^{-2}\left(8.465 \times 10^{-2}\right)$ \\
\hline$\left\{\mathrm{CO}_{2}, \mathrm{CO}\right\}$ & $(0.219,0.293)^{T}$ & $4.464 \times 10^{-2}\left(5.281 \times 10^{-2}\right)$ \\
\hline$\left\{\mathrm{H}_{2} \mathrm{O}, \mathrm{CO}_{2}, \mathrm{CO}, \mathrm{H}_{2}\right\}$ & $(-0.500,0.754,0.814,-0.231)^{T}$ & $4.656 \times 10^{-3}\left(1.066 \times 10^{-1}\right)$ \\
\hline
\end{tabular}

Optimal reaction progress variable for application to diffusion and partially premixed flames; temperature in fuel and oxidizer stream are $298 \mathrm{~K}$ and pressure is 1 bar. 
Table 1 summarizes the results obtained from the optimization problem. The first column shows the set of species that are used to define $C$, the second column shows the weights $\mathbf{w}$ that result in the optimal progress variable, and the last column contains the corresponding value for the cost function. For comparison, cost functions that are evaluated by setting all weights to unity are shown in parentheses.

Focusing first on the methane/air configuration, shown in the uppermost section of Table 1, it can be seen that the optimal progress variable obtained when including only two species in the definition of $C$ yields little improvement in the value of the cost function. Note also that for the first case, the coefficient for $Y_{\mathrm{H}_{2} \mathrm{O}}$ is very small, so that the progress variable is essentially equal to the normalized $\mathrm{CO}_{2}$ mass fraction.

The consideration of four species in the definition of an optimal progress variable provides greater flexibility in adjusting the weight coefficients (compared to the two-species case). In all of the flame configurations considered here, this results in considerably lower values of the cost function and significantly improved optimality of the progress variable. In fact, the value for $\mathcal{L}$ is approximately thirty times smaller compared to the results obtained by only including two species in $\mathcal{S}$, and more than fifty times smaller than the cost function that is obtained by employing Eq. (C2). It is also interesting to point out that for the methane/air diffusion flame considered here, all four weight coefficients are comparable in magnitude; however, the coefficient $w_{\mathrm{H}_{2} \mathrm{O}}$ is negative. While this is a direct result of the optimization procedure and the problem statement, it is noteworthy to point out that the resulting expression for $C$ does not necessarily correspond to the conventional notion that $C$ represents the "progress of reaction."

Similar observations can be made for the other configurations that are presented in Table 1 . These configurations include a partially premixed $\mathrm{CH}_{4}$ /air flame (middle) and a flame operating with a $\mathrm{H}_{2} / \mathrm{CH}_{4}$ fuel mixture (bottom). From this comparison the following conclusions can be drawn. First, it can be seen that each flame configuration requires different values for the weight coefficients $\mathbf{w}$ from which the optimal progress variable is constructed. This in turn suggests that there is no general expression for $C$, and $C$ is dependent on the particular flame configuration. Second, the results also suggest that for the description of simple hydrocarbon-fuels the consideration of the four species contained in the water gas shift reaction provides adequate flexibility in identifying an optimal progress variable.

\section{2. n-Heptane/hydrogen-air combustion system}

The second application considers the combustion of a fuel mixture of $n$-heptane and hydrogen

$$
\alpha \mathrm{C}_{7} \mathrm{H}_{16}+(1-\alpha) \mathrm{H}_{2},
$$

where $\alpha \in[0,1]$ is the fuel mixture ratio. In the following, the parameter $\alpha$ is varied to investigate the effect of the mixture composition on the definition of the reaction progress variable. The oxidizer is air, and pressure and temperature of the reactants are 1 bar and $300 \mathrm{~K}$, respectively. The chemistry is described by the reduced $n$-heptane mechanism due to Seiser et al. [26], consisting of 770 reversible chemical reactions among 159 species.

In this application, two different species sets $\mathcal{S}$ are considered for defining the progress variable. The first set is defined as $\mathcal{S}_{1}=\left\{\mathrm{H}_{2} \mathrm{O}, \mathrm{CO}_{2}, \mathrm{CO}, \mathrm{H}_{2}\right\}$, and is motived from the results of the previous section where it was found that this four-speciesparameterization provides sufficient flexibility for optimizing $C$. The second set contains all species that have a maximum mass fraction larger than a pre-specified threshold of $\xi=10^{-3}: \mathcal{S}_{2}=\left\{Y_{i} \mid \max \left(Y_{i}(Z, \Lambda)\right) \geqslant \xi\right\}$. It is noted that this simple threshold-criterion was selected for illustration purposes, and does not necessarily fulfill principle (b). To resolve this issue, a reaction time-scale analysis could be used to identify species that can be included in the set $\mathcal{S}$.

Results of the parametric investigation are presented in Fig. 1, showing the final value of the cost function versus the $\mathrm{C}_{7} \mathrm{H}_{16} / \mathrm{H}_{2}$-mixing ratio $\alpha$. Results shown in black correspond to the reference case in which the progress variable is defined

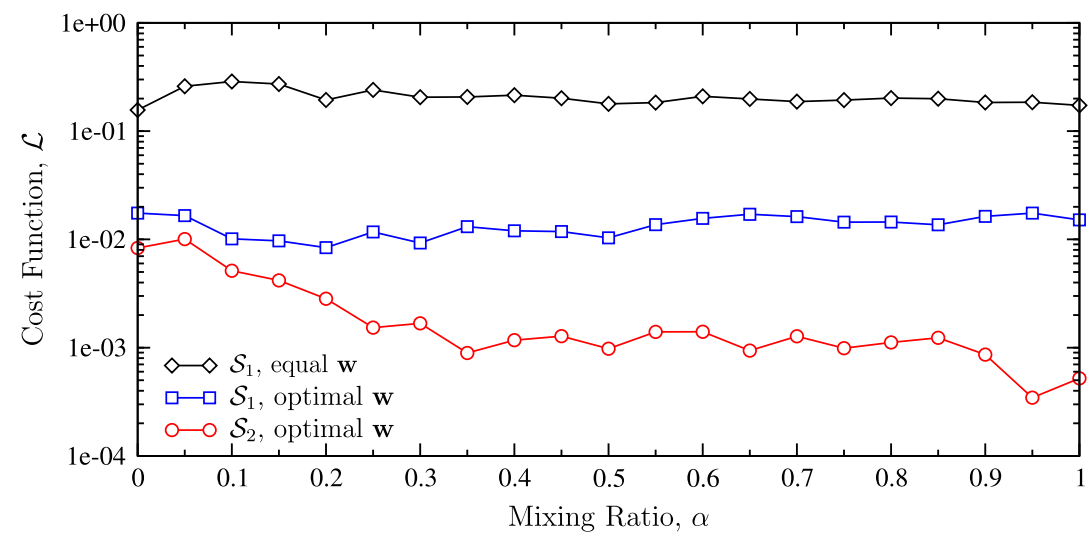

Fig. 1. Comparison of cost function as function of mixing ratio $\alpha$ for an $n$-heptane/hydrogen-air mixture; cost function is evaluated from Eq. (6) with $\Omega=C$ and threshold value $\varepsilon=10^{-10}$. 


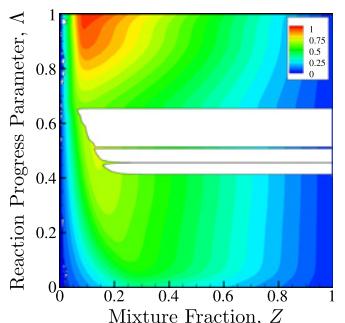

(a) $\mathcal{S}_{1}$, equal $\mathbf{w}$.

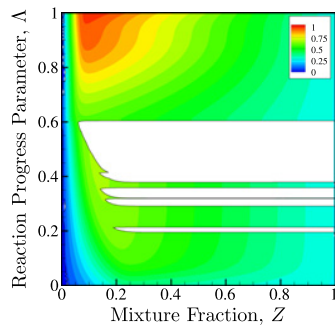

(d) $\mathcal{S}_{1}$, equal $\mathbf{w}$

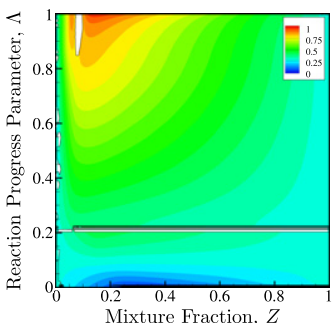

(b) $\mathcal{S}_{1}$, optimal $\mathbf{w}$

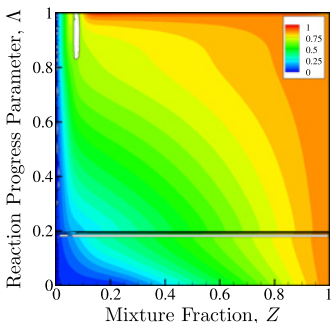

(e) $\mathcal{S}_{1}$, optimal $\mathbf{w}$.

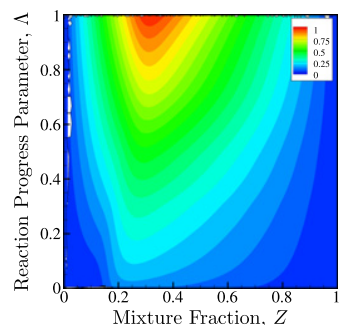

(c) $\mathcal{S}_{2}$, optimal w.

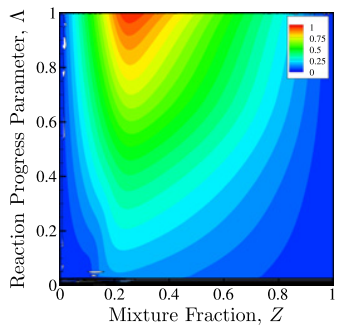

(f) $\mathcal{S}_{2}$, optimal $\mathbf{w}$.

Fig. 2. Comparison of reaction progress variable $C(Z, \Lambda)$ for a $\mathrm{C}_{7} \mathrm{H}_{16} / \mathrm{H}_{2}$-air mixture with $\alpha=1$ (top row) and $\alpha=0.2$ (bottom): progress variable defined from (a,d) the set $\mathcal{S}_{1}$ using equal weighting coefficients, (b,e) the set $\mathcal{S}_{1}$ with optimized weighting coefficients, and (c,f) the extended set $\mathcal{S}_{2}$ and optimized weighting coefficients.

from the set $\mathcal{S}_{1}$ and equal weighting coefficients $\mathbf{w}$. It can be seen that the cost-function (and corresponding specification of $C$ ) is dependent on the mixture composition. The error in representing the compositional state-space using this static progress-variable definition is largest around $\alpha=0.1$ and decreases with increasing $n$-heptane content in the mixture.

By optimizing the weighting coefficients $\mathbf{w}$ for the set $\mathcal{S}_{1}$ (blue line) ${ }^{1}$ significant improvements in the cost function over the entire range of mixture compositions can be obtained. Further improvements of $\mathcal{L}$ by as much as an order of magnitude can be achieved by extending the set of species to $\mathcal{S}_{2}$ (red line). This improvement is particularly pronounced for heptane-rich mixtures, and even for high-hydrogen containing mixtures an optimal selection of weighting coefficients can provide further improvements in the regularization of the progress-variable definition. For the pure $n$-heptane mixture $(\alpha=1)$, it can be seen that the proposed regularization technique in conjunction with the extended species set $\mathcal{S}_{2}$ improves the cost function by more than two orders of magnitude compared to the baseline case.

Comparisons of the three progress variable formulations for $\alpha=1$ and $\alpha=0.2$ are presented in Fig. 2. The blanked-out regions illustrate the state-space composition for which $C$ is non-monotonic in $\Lambda$, corresponding to a non-zero integrand in Eq. (6). This figure shows that the functional form of $C$ and the region of non-monotonicity are dependent on the mixture composition. By comparing the second and the third columns, it can be seen that with increasing flexibility in the problem formulation, the regularization of the progress variable definition is improved.

In this context, it is noted that the regularization method presented in Eq. (7) is formulated as solution to an unconstrained optimization problem. To enforce certain constraints on the definition of $C$ a Lagrangian multiplier,

$$
\iint G(Z, \Lambda) d \Lambda d Z
$$

can be included in the optimization problem. For instance, by defining the integrand as $G=\partial_{\Lambda} C-1$ an orthogonality condition between $Z$ and $C$ can be introduced. Similarly, by minimizing the source term of the progress variable through the constraint $G=\left|\dot{\omega}_{C}\right|$, a second conserved scalar variable $C$ can be identified that is independent from $Z$. So far, such model extensions have not been considered, but can provide additional flexibilities towards improving the regularity of the reacting progress variable.

\section{Conclusions}

Reaction progress variables used in combustion models are often defined in an ad hoc way. They are, therefore, seldom "optimal" and can potentially produce inaccurate predictions when applied in combustion simulations. This paper identifies four optimality conditions to guide the selection of a reaction progress variable and proposes a method to optimize the progress variable with respect to these conditions. The method is consistent with many previous progress-variable

\footnotetext{
1 For interpretation of color in Fig. 1, the reader is referred to the web version of this article.
} 
formulations. The optimization introduces negligible overhead since it is performed in a preprocessing step prior to the chemistry tabulation. The only required input from the user is the specification of a set of species from which the progress variable is constructed.

The selection of species has obvious implications on the accuracy of the final model and the amount of improvement that can be expected during optimization. Future implementations of this method could incorporate a time-scale analysis to automatically identify a set of species as an early step in the algorithm. For example, information from a computational singular perturbation (CSP) of the underlying kinetic mechanism could be used to guide the selection of candidate species in the definition of the progress variable. The "radical pointer" criteria $[18,19]$ that CSP uses to eliminate species that are exhausted in fast reaction modes could be a useful indicator. Species that CSP identifies as important in different time epochs would then be a natural choice for inclusion in progress variables that are tuned to a particular reaction time scale. These extensions are left for future investigations.

The potential of this method was demonstrated in applications to partially-premixed and diffusion flames. It is noted that the proposed method is equally applicable to combustion models in which the thermochemical state-space is constructed from premixed flamelets, and can also be extended to combustion problems involving multiple reactant streams.

The proposed method can lead to dramatic improvements in the definition of a regularized progress variable and alleviates the need for expert knowledge to identify such a quantity. This appears to be of particular relevance since it is shown that the definition of an optimal progress variable is dependent on the reaction chemistry, combustion mode, and fuel composition.

\section{Acknowledgments}

Financial support through the Air Force Office of Scientific Research under Award No. FA9550-11-1-0031 is gratefully acknowledge.

\section{References}

[1] T. Lu, C.K. Law, Toward accommodating realistic fuel chemistry in large-scale computations, Prog. Energ. Combust. Sci. 35 (2009) $192-215$.

[2] N. Peters, Local quenching due to flame stretch and non-premixed turbulent combustion, Combust. Sci. Tech. 30 (1983) 1-17.

[3] N. Peters, Laminar diffusion flamelet models in non-premixed turbulent combustion, Prog. Energ. Combust. Sci. 10 (3) (1984) 319-339.

[4] U. Maas, S.B. Pope, Simplifying chemical kinetics: intrinsic low-dimensional manifolds in composition space, Combust. Flame 88 (3-4) (1992) 239-264.

[5] O. Gicquel, N. Darabiha, D. Thevenin, Laminar premixed hydrogen/air counterflow flame simulations using flame prolongation of ILDM with differential diffusion, Proc. Combust. Inst. 28 (2000) 1901-1908.

[6] J.A. van Oijen, L.P.H. de Goey, Modelling of premixed laminar flames using flamelet-generated manifolds, Combust. Sci. Tech. 161 (2000) $113-137$.

[7] C.D. Pierce, P. Moin, Progress-variable approach for large-eddy simulation of non-premixed turbulent combustion, J. Fluid Mech. 504 (2004) $73-97$.

[8] M. Ihme, C.M. Cha, H. Pitsch, Prediction of local extinction and re-ignition effects in non-premixed turbulent combustion using a flamelet/progress variable approach, Proc. Combust. Inst. 30 (2005) 793-800.

[9] J.A. van Oijen, F.A. Lammers, L.P.H. de Goey, Modeling of complex premixed burner systems by using flamelet-generated manifolds, Combust. Flame 127 (3) (2001) 2124-2134.

[10] J.A. van Oijen, L.P.H. de Goey, Modelling of premixed counterflow flames using the flamelet-generated manifold method, Comb. Theory Model. 6 (2002) 463-478.

[11] B. Fiorina, R. Baron, O. Gicquel, D. Thevenin, S. Carpentier, N. Darabiha, Modelling non-adiabatic partially premixed flames using flame-prolongation of ILDM, Combust. Theory Model. 7 (2003) 449-470.

[12] B. Fiorina, O. Gicquel, L. Vervisch, S. Carpentier, N. Darabiha, Approximating the chemical structure of partially premixed and diffusion counterflow flames using FPI flamelet tabulation, Combust. Flame 140 (2005) 147-160.

[13] B. Fiorina, O. Gicquel, L. Vervisch, S. Carpentier, N. Darabiha, Premixed turbulent combustion modeling using tabulated detailed chemistry and PDF, Proc. Combust. Inst. 30 (2005) 867-874.

[14] M. Ihme, H. Pitsch, Prediction of extinction and reignition in non-premixed turbulent flames using a flamelet/progress variable model. 1. A priori study and presumed PDF closure, Combust. Flame 155 (2008) 70-89.

[15] M. Ihme, H. Pitsch, Prediction of extinction and reignition in non-premixed turbulent flames using a flamelet/progress variable model. 2. A posteriori study with application to Sandia flames D and E, Combust. Flame 155 (2008) 90-107.

[16] M. Ihme, Pollutant formation and noise emission in turbulent non-premixed flames, Ph.D. thesis, Stanford University, 2007.

[17] E. Knudsen, H. Pitsch, A general flamelet transformation useful for distinguishing between premixed and non-premixed modes of combustion, Combust. Flame 156 (2009) 678-696.

[18] S.H. Lam, Using CSP to understand complex chemical kinetics, Combust. Sci. Tech. 89 (5-6) (1993) 375-404.

[19] S.H. Lam, D.A. Goussis, The CSP method for simplifying kinetics, Int. J. Chem. Kinet. 26 (1994) 461-486.

[20] R.M. Lewis, V. Torczon, Pattern search algorithms for bound constrained minimization, SIAM J. Optim. 9 (4) (1999) 1082-1099.

[21] V. Torczon, On the convergence of pattern search algorithms, SIAM J. Optim. 7 (1) (1997) 1-25.

[22] C. Audet, J.E. Dennis Jr. Analysis of generalized pattern searches, SIAM J. Optim. 13 (3) (2003) 889-903.

[23] H. Pitsch, FlameMaster v3.1: A C++ computer program for 0D combustion and 1D laminar flame calculations, 1998.

[24] N. Peters, Turbulent Combustion, Cambridge University Press, Cambridge, 2000.

[25] C.T. Bowman, R.K. Hanson, D.F. Davidson, W.C. Gardiner, V. Lissianski, G.P. Smith, D.M. Golden, M. Frenklach, M. Goldenberg, GRI-Mech 2.11, 1997. available from http:<http://www.me.berkeley.edu/gri-mech/>.

[26] R. Seiser, H. Pitsch, K. Seshadri, W.J. Pitz, H.J. Curran, Extinction and autoignition of $n$-heptane in counterflow configuration, Proc. Combust. Inst. 28 (2000) 2029-2037. 